pressure limits, together with the change in character of the ranges obtained.

We wish to express our thanks to Dr. D. T. A Townend for interesting suggestions made during our visit to the British Coal Utilization Research Associa. tion, Leatherhead, last September.

This investigation is being continued in this Laboratory and will be described fully later. R. DElBOURGo P. Laffitte

Laboratoire de Chimie Générale, Faculté des Sciences, Paris.

Feb. 6.

1 Smith, E. C. W., Proc. Roy. Soc., A, 174, 110 (1940),

\section{g-Factors of Binary Ferrites at Low Temperature}

A PHYSICAL theory of the magnetic properties of ferrites has recently been given by $\mathrm{Née}^{1}$. Experimental investigations by which this theory may be tested include those of $\mathrm{E}$. W. Gorter ${ }^{2}$ on the saturation magnetization of a number of simple and mixed ferrites at low temperature, and of $\mathrm{H}$. $\mathrm{G}$. Beljers and $D$. Polder ${ }^{3}$ on the $g$-factors of nickel-zine ferrites of various compositions at room temperature.

It is desirable to determine the $g$-factors of these materials at the same low temperatures at which the saturation magnetization has been measured. In the present work, $g$-factors have been obtained for various ferrites by a resonance method over a temperature range of $0^{\circ} \mathrm{C}$. to $-195^{\circ} \mathrm{C}$., using spherical specimens $2 \mathrm{~mm}$. in diameter, and a wave-length of $3.2 \mathrm{~cm}$. The results for the two extreme temperatures are shown in the accompanying table.

g-FACTORS OF BINARY FERRITES

Composition: $x M_{a} \mathrm{Fe}_{2} \mathrm{O}_{6}+(1-x) M_{b} \mathrm{Fe}_{2} \mathrm{O}_{4}$

(i) $M a=Z n ; M b=\mathrm{Ni}$.

\begin{tabular}{|c|cc|cc|cc|}
\hline & \multicolumn{2}{|c|}{$\mathrm{Ni}$} & \multicolumn{2}{|c|}{$\mathrm{Cu}$} & \multicolumn{2}{|c|}{$\mathrm{Mg}$} \\
\hline $0 \cdot 0$ & $0^{\circ}$ & $-195^{\circ}$ & $0^{\circ}$ & $-195^{\circ}$ & $0^{\circ}$ & $-195^{\circ}$ \\
$0 \cdot 2$ & $2 \cdot 26$ & $2 \cdot 39$ & $2 \cdot 09$ & $2 \cdot 32$ & $2 \cdot 04$ & $2 \cdot 24$ \\
$0 \cdot 4$ & $2 \cdot 40$ & $2 \cdot 69$ & $2 \cdot 10$ & $2 \cdot 33$ & $2 \cdot 06$ & $2 \cdot 37$ \\
$0 \cdot 6$ & $2 \cdot 40$ & $2 \cdot 83$ & $2 \cdot 21$ & $2 \cdot 47$ & 2.07 & $2 \cdot 44$ \\
$0 \cdot 8$ & $2 \cdot 40$ & $2 \cdot 69$ & $2 \cdot 11$ & $2 \cdot 44$ & 2.05 & $2 \cdot 53$ \\
$1 \cdot 0$ & 1.98 & $2 \cdot 16$ & 1.95 & $2 \cdot 07$ & 1.93 & $2 \cdot 11$ \\
\hline
\end{tabular}

(ii) $M a=\mathrm{Mg} ; M o=\mathrm{Ni}$.

\begin{tabular}{|c|cc|cc|}
\hline$x$ & & & & \\
& $0^{\circ}$ & $-195^{\circ}$ & $0^{\circ}$ & $-195^{\circ}$ \\
\hline $0 \cdot 0$ & $2 \cdot 26$ & $2 \cdot 39$ & $2 \cdot 09$ & $2 \cdot 32$ \\
$0 \cdot 2$ & $2 \cdot 17$ & $2 \cdot 32$ & $2 \cdot 09$ & $2 \cdot 37$ \\
$0 \cdot 4$ & $2 \cdot 17$ & $2 \cdot 17$ & $2 \cdot 11$ & $2 \cdot 35$ \\
0.6 & $2 \cdot 11$ & $2 \cdot 11$ & $2 \cdot 11$ & $2 \cdot 24$ \\
0.8 & $2 \cdot 09$ & $2 \cdot 16$ & $2 \cdot 11$ & $2 \cdot 27$ \\
1.0 & $2 \cdot 04$ & $2 \cdot 24$ & $2 \cdot 04$ & $2 \cdot 24$ \\
\hline
\end{tabular}

For group (i), the $g$-factor increases at first with increasing content of zinc ferrite, and it passes through a maximum in a composition-range approximately the same as that for which Gorter's curves give a maximum for the saturation magnetization. For the $\mathrm{Ni}-\mathrm{Mg}$ ferrite in group (ii), the $g$-value decreases monotonically with content of magnesiumferrite at $0^{\circ} \mathrm{C}$.; but at $-195^{\circ} \mathrm{C}$. there is a minimum, which has also been observed ${ }^{4}$ in the saturation magnetization. For the copper-magnesium ferrite, the $g$-value is practically constant at $0^{\circ} \mathrm{C}$. The maximum at $-195^{\circ} \mathrm{C}$. has also been found ${ }^{4}$ in the saturation magnetization.

A theoretical discussion of these results in the light of Néel's theory, and details of the apparatus, procedure and of the methods of preparation of the specimens, will be published in Sci. Rep. RITU.

Tosihiko OKamura YosIharu TORIZUKA

Research Institute for Scientific Measurement, Sendai.

Jan. 19.

${ }^{1}$ Néel, L., Ann. Phys., 3, 137 (1948).

2 Gorter, E. W., Nature, 165, 798 (1950).

${ }^{3}$ Beljers, H. G., and Polder, D., Nature, 165, 800 (1950).

${ }^{4}$ Okamura, T., and Simoizaka, J., Sci. Rep. RITU. (in the press).

\section{Triphenylchloroethylene, Tritylmethyl Chloride and Related Halides}

Trityluerhyl chloride, $\mathrm{Ph}_{3} \mathrm{C} . \mathrm{CH}_{2}$. $\mathrm{Cl}$, has interesting structural features, especially from the point of view of investigations of reaction mechanism ${ }^{1}$. We wish to record briefly the preparation, properties and reactions of this compound, and to refer to some related halides. The substance previously listed in the literature ${ }^{2}$ as tritylmethyl chloride is triphenylchloroethylene, $\mathrm{Ph}_{2} \mathrm{C}=\mathrm{C}(\mathrm{Cl}) \mathrm{Ph}$.

'I ritylmethyl chloride (m.p. $101 \cdot 5^{\circ}$ ) was obtained in good yield from triphenylmethyl sodium and methylene chloride in ethereal solution. Tritylmethyl bromide (m.p. $93^{\circ}$ ) was prepared similarly, but in poor yield. An attempt to obtain the iodide (see below) by the same method gave triphenylmethane and triphenylethylene. From $\mathrm{Cl}\left(\mathrm{CH}_{2}\right)_{2} \mathrm{Cl}$ and $\mathrm{Cl}\left(\mathrm{CH}_{2}\right)_{3} \mathrm{Br}$, $\beta$-tritylethyl and $\gamma$-tritylpropyl chloride (m.p. $113^{\circ}$ and $130^{\circ}$, respectively) have been similarly obtained. Their reactions are being examined, together with those of tritylmethyl bromide and iodide.

The following observations refer to tritylmethyl chloride. The ultra-violet absorption spectrum was found to be similar to that of 1,1,1-triphenylethane. With the Grignard reagent, water gave 1,1,l-triphenylethane, bromine gave tritylmethyl bromide, and iodine gave a rather unstable iodide (m.p. 101', with decomp.) regarded provisionally as 'tritylmetizyl iodide'. The reaction with sodium in liquid ammonia, followed by treatment with ammonium chloride, yielded 1,1,1-triphenylethane. (All the new compounds, the names of which are italicized above, gave correct analytical data.)

At temperatures in the neighbourhood of its melting point, a slow decomposition of the chloride takes place, giving hydrogen chloride and triphenylethylene. This change is powerfully catalysed by certain metallic compounds, ferric and stannic chlorides being particularly active. Interaction with benzene in the presence of aluminium chloride gave sym.tetraphenylethane. The reaction with sodium appears to be tardy, and the thermal decomposition of the chloride is a complication; in petroleum solution at $120^{\circ}$, triphenylethylene was isolated from the products. A preliminary examination of the reactions with Grignard reagents in the presence of cobaltous chloride yielded indefinite results.

Tritylmethyl chloride exhibits very marked steric hindrance in bimolecular nucleophilic replacements $\left(S_{N} 2\right)$. The reactions in hydroxylic solvents are not of this type: they are fairly facile, their rates are 